\title{
A Comparative Study between X_Lets Family for Image Denoising
}

\author{
Beladgham Mohamed ${ }^{1}$, Habchi Yassine ${ }^{2}$, Moulay Lakhdar Abdelmouneim ${ }^{3}$, Abdesselam Bassou ${ }^{4}$ \\ , Taleb-Ahmed Abdelmalik ${ }^{5}$ \\ ${ }^{1}$ University of Bechar, Algeria, \\ Department of Electronic, Faculty of Sciences and Technology, \\ Postal address, 08000, Bechar, Algeria, E-Mail: beladgahm.tlm@gmail.com \\ ${ }^{2}$ University of Bechar, Algeria, \\ Department of Electronic, Faculty of Sciences and Technology, \\ Postal address, 08000, Bechar, Algeria, E-Mail: habchi8article@gmail.com \\ ${ }^{3}$ University of Bechar, Algeria, \\ Department of Electronic, Faculty of Sciences and Technology, \\ Postal address, 08000, Bechar, Algeria, E-Mail: moulaylakhdar78@yahoo.fr \\ ${ }^{4}$ University of Bechar, Algeria, \\ Department of Electronic, Faculty of Sciences and Technology, \\ Postal address, 08000, Bechar, Algeria, E-mail: a.bassou@gmail.com \\ ${ }^{5}$ Biomecanic Laboratory, Valencienne University, France \\ 59313, Valenciennes, Francia, E-Mail: taleb@univ-valenciennes.fr
}

\begin{abstract}
Research good representation is a problem in image processing for this, our works are focused in developing and proposes some new transform which can represent the edge of image more efficiently, Among these transform we find the wavelet and ridgelet transform these both types transforms are not optimal for images with complex geometry, so we replace this two types classical transform with other effectiveness transform named bandelet transform, this transform is appropriate for the analysis of edges of the images and can preserve the detail information of high frequency of noisy image. De-noising is one of the most interesting and widely investigated topics in image processing area. In order to eliminate noise we exploit in this paper the geometrical advantages offered by the bandelet transform to solve the problem of image de-noising. To arrive to determine which type transform allows us high quality visual image, a comparison is made between bandelet, curvelet, ridgelet and wavelet transform, after determining the best transform, we going to determine which type of image is adapted to this transform. Numerically, we show that bandelet transform can significantly outperform and gives good performances for medical image type TOREX, and this is justified by a higher PSNR value for gray images.
\end{abstract}

Index Terms - Bandelet transform, Contourlet transform, Curvelet transform, Ridgelet transform, Quadtree segmentation.

\section{INTRODUCTION}

Image de-noising is one of the most popular research fields in image processing due to the fact that it is extremely difficult to form a general global de-noising scheme effective for all types of noise as well as all types of images. A common problem in image denoising is the blurring of the prominent edges in the image which can cause discrepancies when the denoising operation is combined with other operations such as image edge detection and segmentation. For image de-noising processing many classical methods can be applied to de-noise, such as wavelet has become a useful tool for analysis of many kinds of problems, wavelets are good at catching point singularities, but for linear singularity, wavelets transform cannot capture well. To overcome the weakness of wavelets in higher dimensions, Candes and Donoho [1] pioneered a new system of representations named ridgelets which they showed to deal effectively with line singularities in 2D, ridgelet [2] is a new perfect tool to analyze and synthesize the 2-dimensional and the high-dimensional singularities.

In the recent years, S.Mallat and E.Le Pennec proposed new transform named bandelet transform [3] construct a basis with a multiscale geometry adapted to image, can capture directional geometric image regularity and provide an optimal approximation for a more complex class of geometric images. 


\section{BANDELET TRANSFORM}

Bandelet transform, introduced by E. Le Pennec and S. Mallat [4] built a base adapted to the geometric content of an image.

The bandelets are obtained from a local deformation of space to align the direction of regularity with a fixed direction (horizontal or vertical) and is reduced to a separable basis [5].
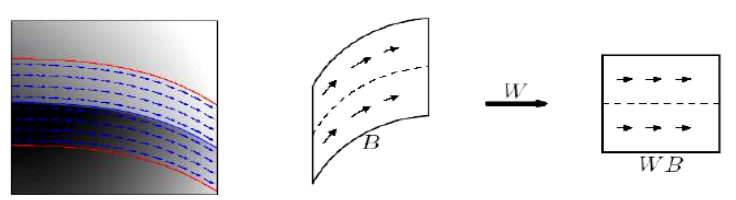

Figure 1. Example of model horizon and deformation of the field according to a geometric flow

\section{A. FLOW-CURVE RELATIONSHIP}

There is a constant correspondence between the flow along the vertical direction and curves whose tangent is never vertical; the flow associated with this curve is given by flow:

$$
\tau(x)=\frac{1}{\sqrt{1+\left|c^{\prime}(x)\right|^{2}}}\left(\begin{array}{l}
1 \\
c^{\prime}(x)
\end{array}\right)
$$

$c^{\prime}(x):$ Slope of optical flow.

We can generate the basic test bandelet according to the flow and geometric regularity of each sub-block. If there is no flow geometry in the sub-blocks, this means that the sub-block is uniformly regular so that we can use the classical separable wavelet basis for treating this sub-block. If not, the sub-block must be processed by the bandelet.

Also, the variation along geometric flow defined in the sub-block means that we can deform the sub-block in horizontal or vertical direction, in this case we can say that the sub-block is uniformly regular.

Calculates the Lagrangian allows us to determine subblock that contains the singularity [6].

$$
L(f, R, B)=\left\|f-f_{R}\right\|^{2}+\lambda \cdot T^{2} \sum_{j}\left(R_{j G}+R_{j B}\right)
$$

\section{$\lambda$ : Lagrangian multiplier.}

$T$ : Quantifiction step.

$R_{j G}$ : is the number of bits to code the optical flew in each square.

$R_{j B}:$ is the number of bits to code the quantized bandelet coefficients.

$f_{R}$ : is the reconstructed $1 \mathrm{D}$ signal by thresholding the coefficients smaller than $T$. The optimal direction in each dyadic sub-region can be defined as $\theta_{\text {opt }}=\arg _{\theta} \min L(f, \theta, T)$

Then the corresponding 1D wavelet coefficients to $\theta_{\text {opt }} \mathrm{t}$ is defined as bandelet coefficients and the optimal direction $\theta_{o p t} \mathrm{t}$ is recorded for reconstructing image. [7]

\section{B. QUADTREE SEGMENTATION}

Segmentation operation it is a division of image space that allows us to have a set of sub-blocks.

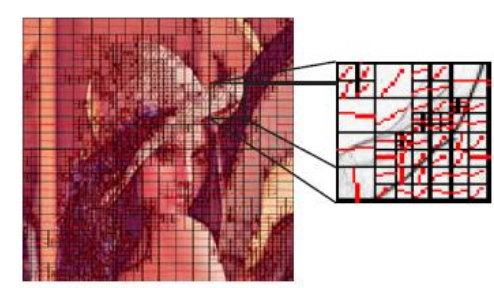

Figure 2. Example of quadtree segmentation

The operating diagram of the quadtree segmentation is the following.

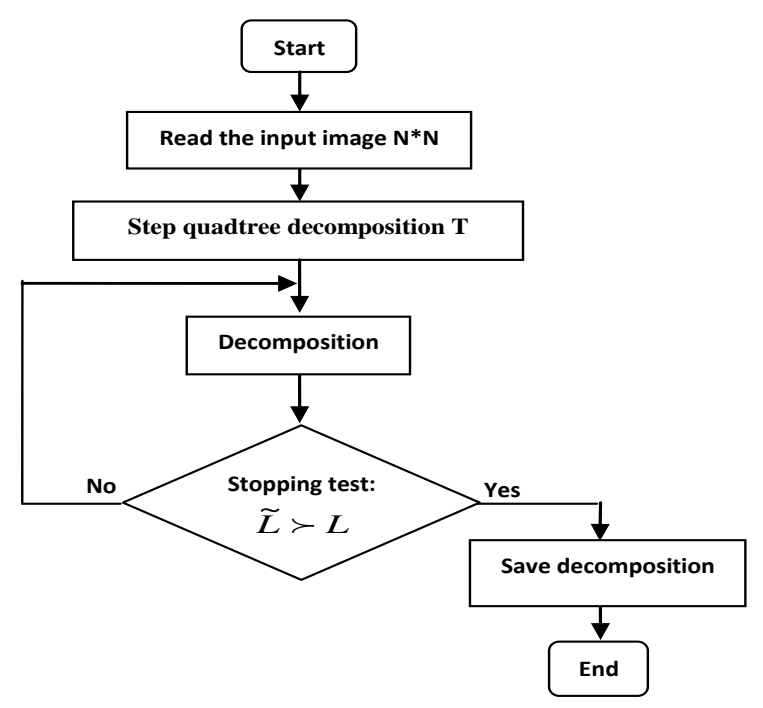

Figure 3. Segmentation quadtree diagram

The optimal segmentation is defined with [8]:

$$
L_{0}(S)=\min \left\{L_{0}(S), \widetilde{L}(S)\right\}
$$

Where

$\tilde{L}(S)=L_{0}\left(S_{1}\right)+L_{0}\left(S_{2}\right)+L_{0}\left(S_{3}\right)+L_{0}\left(S_{4}\right)+L_{0}(S)+\lambda \cdot T^{2}$

$L_{0}\left(S_{i}\right)$ : Lagrangian of sub-blocks.

\section{THE OPERATOR OF DEFORMATION}

The deformation operation is a local operation on a block that contains a curve singularity to align or correct in a direction horizontal and vertical. 


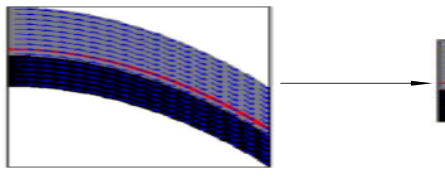

(a)

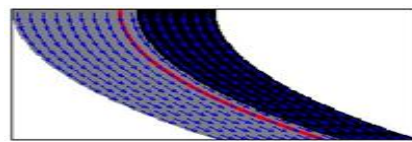

(c) (b)

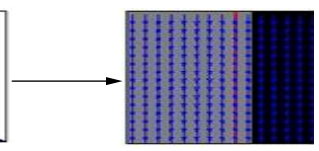

(d)

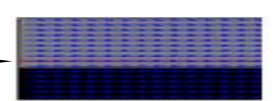

Figure 4. Segmentation Example of model horizon and deformation of the field according to a geometric flow. Image distortion: (a) an image having a horizontal flow, (b) its image by the operator W, (c) an image having a vertical flow, (d) the image by the operator $\mathrm{W}$

Note that the two-dimensional Wavelet basis is:

$$
\left\{\begin{array}{l}
\phi_{j, n_{1}}\left(x_{1}\right) \psi_{j, n_{2}}\left(x_{2}\right) \\
\psi_{j, n_{1}}\left(x_{1}\right) \phi_{j, n_{2}}\left(x_{2}\right) \\
\psi_{j, n_{1}}\left(x_{1}\right) \psi_{j, n_{2}}\left(x_{2}\right)
\end{array}\right\}
$$

Deformation operation gives a wavelet orthonormal basis of $L^{2}(\Omega)$ :

$$
\left\{\begin{array}{l}
\phi_{j, n_{1}}\left(x_{1}\right) \psi_{j, n_{2}}\left(x_{2}-c\left(x_{1}\right)\right) \\
\psi_{j, n_{1}}\left(x_{1}\right) \phi_{j, n_{2}}\left(x_{2}-c\left(x_{1}\right)\right) \\
\psi_{j, n_{1}}\left(x_{1}\right) \psi_{j, n_{2}}\left(x_{2}-c\left(x_{1}\right)\right)
\end{array}\right\}
$$

The vertical $\psi_{j, n}^{V}$ and diagonal $\psi_{j, n}^{D}$ Wavelets have vanishing moments along contour, they are therefore suitable for the approximation of function $f$ is regular along contour is not the case of horizontal wavelet $\psi_{j, n}^{H}$, to be replaced by new functions:

$\psi_{l, n_{1}}\left(x_{1}\right) \psi_{j, n_{2}}\left(x_{2}-c\left(x_{1}\right)\right)$

This is called bandeletization and we check it is implemented by a simple 1D discrete Wavelet transform [9], [10].

The orthonormal basis of bandelet of field warping is defined by:

$\left.\left(\begin{array}{l}\psi_{l, n_{1}}\left(x_{1}\right) \psi_{j, n_{2}}\left(x_{2}-c\left(x_{1}\right)\right) \\ \psi_{j, n_{1}}\left(x_{1}\right) \phi_{j, n_{2}}\left(x_{2}-c\left(x_{1}\right)\right) \\ \psi_{j, n_{1}}\left(x_{1}\right) \psi_{j, n_{2}}\left(x_{2}-c\left(x_{1}\right)\right)\end{array}\right)=\left\{\begin{array}{l}\psi_{l, n}^{H} \\ \psi_{j, n}^{V} \\ \psi_{j, n}^{D}\end{array}\right\} j, l\right\rangle n_{1}, n_{2}$

The orthogonality property is not sufficient; we take the bandelet dimensional biorthogonal $\psi$ and its scaling function ${ }^{\phi}$, the same construction gives a biorthogonal basis of $L^{2}(\Omega)$.

\section{DIAGRAM OF OPERATION}

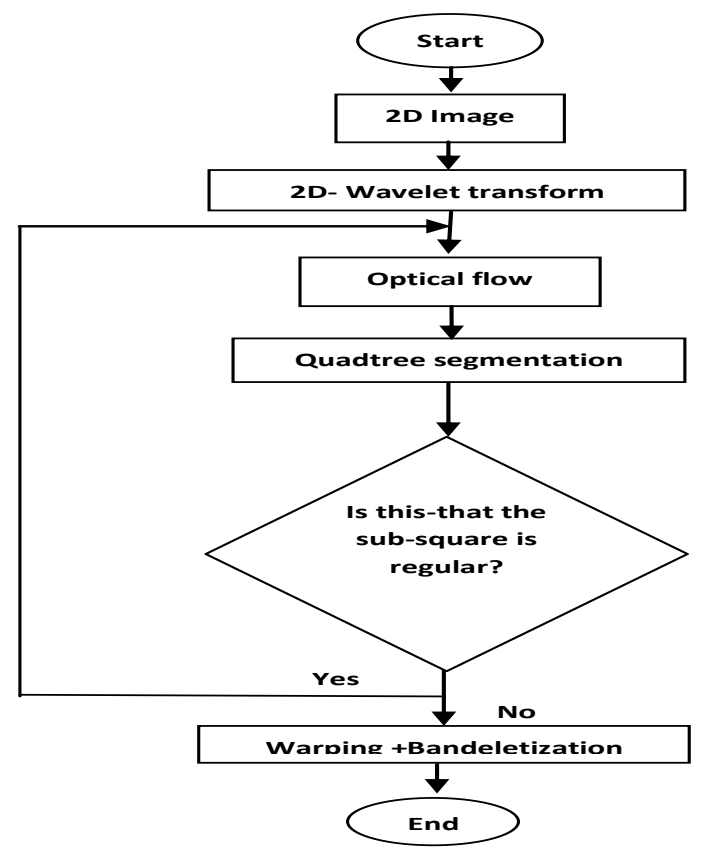

Figure 5. Bandelet transform operation diagram

\section{IV.QUALITYEVALUATION PARAMETER}

The Peak Signal to Noise Ratio (PSNR) is the most commonly used as a measure of quality of reconstruction in image compression. The PSNR were identified using the following formulate:

$$
M S E=\frac{1}{M \cdot N} \cdot \sum_{i=0}^{M-1 N-1} \sum_{j=0}[I(i, j)-\hat{I}(i, j)]^{2}
$$

Mean Square Error (MSE) which requires two MxN gray scale images $I$ and $\hat{I}$ where one of the images is considered as a compression of the other is defined as:

- The PSNR is defined as:

$P S N R=10 \cdot \log _{10} \frac{\left(2^{R}-1\right)^{2}}{M S E}[d B]$

Usually an image is encoded on 8 bits. It is represented by 256 gray levels, which vary between 0 and 255, the extent or dynamics of the image is 255 .

\section{- The structural similarity index (SSIM):}

The PSNR measurement gives a numerical value on the damage, but it does not describe its type. Moreover, as is often noted in [11], [12], [13], it does not quite 
represent the quality perceived by human observers. For medical imaging applications where images are degraded must eventually be examined by experts, traditional evaluation remains insufficient. For this reason, objective approaches are needed to assess the medical imaging quality. We then evaluate a new paradigm to estimate the quality of medical images, specifically the ones compressed by wavelet transform, based on the assumption that the human visual system (HVS) is highly adapted to extract structural information. The similarity compares the brightness, contrast and structure between each pair of vectors, where the structural similarity index (SSIM) between two signals $\mathrm{x}$ and $\mathrm{y}$ is given by the following expression:

$$
\operatorname{SSIM}(x, y)=l(x, y) c(x, y) s(x, y)
$$

Finally the quality measurement can provide a spatial map of the local image quality, which provides more information on the image quality degradation, which is useful in medical imaging applications. For application, we require a single overall measurement of the whole image quality that is given by the following formula:

$$
\operatorname{MSSIM}(I, \hat{I})=\frac{1}{M} \sum_{i=1}^{M} \operatorname{SSIM}\left(I_{i}, \hat{I}_{i}\right)
$$

Where $I$ and $\hat{I}$ are respectively the reference and degraded images, ${ }{ }_{i}$ and $\hat{I}_{i}$ are the contents of images at the i-th local window.

$\mathrm{M}$ : the total number of local windows in image. The MSSIM values exhibit greater consistency with the visual quality.

\section{- CORRELATION COEFFICIENT (CC)}

The correlation coefficient, $r$, is widely used in statistical analysis, and image processing for compared two images of the same object (or scene). The $r$ value indicates whether the object has been altered or moved. The correlation coefficient is defined as

$$
r=\frac{\sum_{i}\left(x_{i}-x_{m}\right)\left(y_{i}-y_{m}\right)}{\sqrt{\sum_{i}\left(x_{i}-x_{m}\right)^{2}} \sqrt{\sum_{i}\left(y_{i}-y_{m}\right)^{2}}}
$$

Where $x_{i}$ is the intensity of the ith pixel in image 1, $y_{i}$ is the intensity of the ith pixel in image $2, x_{m}$ is the mean intensity of image 1 , and $y_{m}$ is the mean intensity of image 2 .

The correlation coefficient has the value $r=1$ if the two images are absolutely identical, $r=0$ if they are completely uncorrelated, and $r=-1$ if they are completely anti-correlated. [14].

\section{RESULTS AND DISCUSSION}

In this article, we give numerical experiments to test our denoising method. For this reason we opted for a set of medical images (MRI, CT, ECHO and TOREX) gray scale coded on 8 bits per pixel. These images are taken from the GE Medical System (database) [15]. The importance of our work lies in the possibility of reducing the noise for which the image quality remains acceptable. Estimates and judgments of the de-noised image quality are given by the PSNR evaluation parameters and the MSSIM similarity Index.

For each application we vary sigma value 0.06 to 0.4 and calculate the PSNR and MSSIM. The results obtained are given in Table1 and Table2.

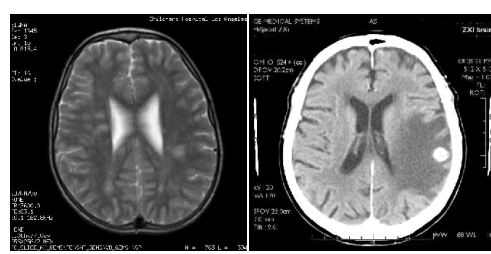

(a)

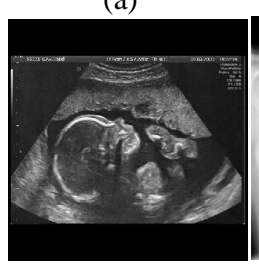

(c) (b)

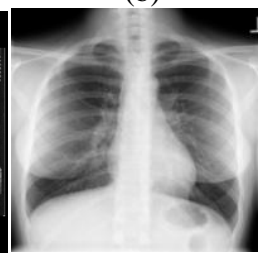

(d)
Figure 6. Popular test images

(a) MRI, (b) CT, (c) ECHO, (d) TOREX

Here, we adopt test image MRI of size $512 \times 512$ encoded by 8 bits per pixel, and with quadtree quantization $\mathrm{T}=10$, the experimental of this results are compared with other value of quadtree quantization $\mathrm{T}=30$. The experimental results are shown in table 1 .

\begin{tabular}{|c|c|c|c|c|c|}
\hline \multicolumn{2}{|c|}{ STEP (T) Sigma } & 0.06 & 0.08 & 0.1 & 0.4 \\
\hline \multirow{2}{*}{$\stackrel{\ominus}{\operatorname{II}}$} & $\begin{array}{c}\text { PSNR } \\
\text { (dB) }\end{array}$ & $\begin{array}{l}29.86 \\
66\end{array}$ & 28.1657 & $\begin{array}{l}26.841 \\
8\end{array}$ & $\begin{array}{l}17.484 \\
6\end{array}$ \\
\hline & MSSIM & $\begin{array}{l}0.783 \\
5\end{array}$ & 0.7405 & 0.7158 & 0.4784 \\
\hline \multirow{2}{*}{ 突 } & $\begin{array}{c}\text { PSNR } \\
\text { (dB) }\end{array}$ & $\begin{array}{l}31.25 \\
75\end{array}$ & 29.5726 & $\begin{array}{l}28.085 \\
9\end{array}$ & $\begin{array}{l}18.031 \\
4\end{array}$ \\
\hline & MSSIM & $\begin{array}{l}0.812 \\
6\end{array}$ & 0.7783 & 0.7503 & 0.5214 \\
\hline
\end{tabular}

TABLE 1. NUMERICAL RESULTS OBTAINED AFTER MRI IMAGE DE-NOISING FOR T=10 AND T=30 


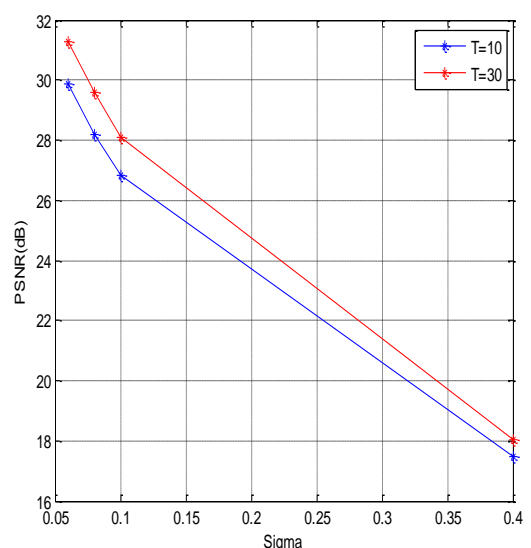

(a) PSNR Variation

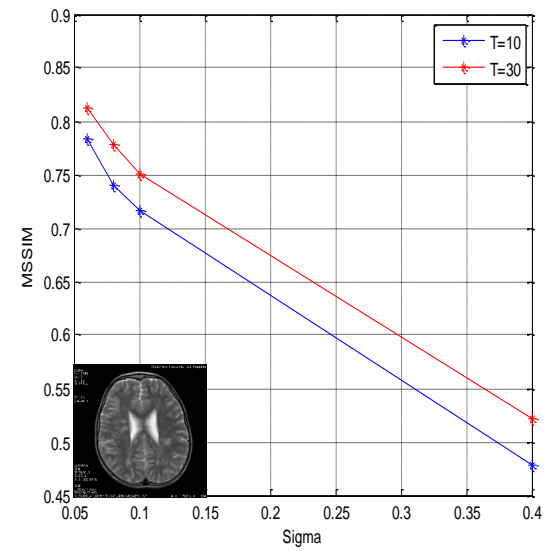

(b) MSSIM Variation

Figure 7. Comparisons of MRI denoising image using bandelet transform between $\mathrm{T}=10$ to $\mathrm{T}=30$

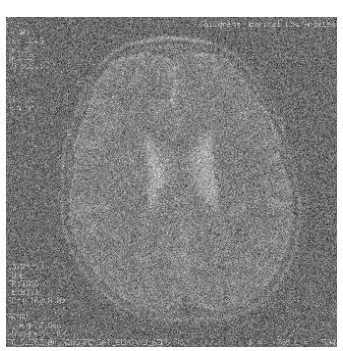

(a)

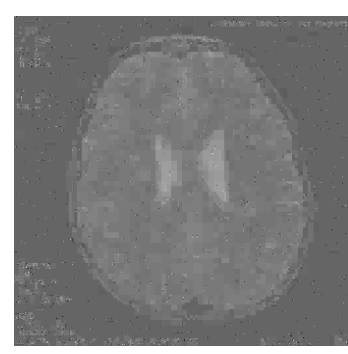

(b)

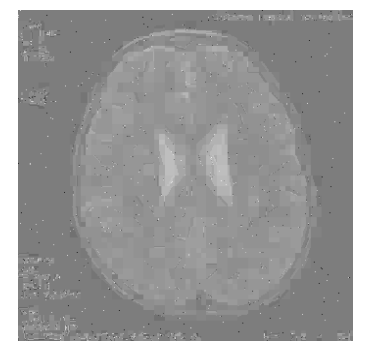

(c)

Figure 8. Denoising MRI images for different values of quadtree quantization $\mathrm{T}$ : (a) Noise image, (b) De-noising image for $\mathrm{T}=10$, (c) De-noising image for $\mathrm{T}=30$.

It can be seen from Table 1, that our algorithm presents excellent de-noising performance for $\mathrm{T}=30$, Fig

8. (c), and it also marks that the PSNR decreases and this can be explained by increasing of sigma value which represents the noise variation that any role that degrade the quality visual of image.

After showed the effectiveness of our algorithm for $\mathrm{T}$ $=30$, this value is fixed in the following work. We have extended this study to a set of medical images, to demonstrate the effectiveness of bandelet transform, we vary the noise variance (sigma) and calculating the evaluation parameters (PSNR, MSSIM); we obtain the following results (Table 2):

TABLE 2. NUMERICAL RESULTS OBTAINED AFTER CT, ECHO AND TOREX IMAGE DE-NOISING FOR T $=30$

\begin{tabular}{|c|c|c|c|c|c|c|}
\hline \multirow{3}{*}{ } & \multicolumn{6}{|c|}{ Image } \\
\hline & \multicolumn{2}{|c|}{$C T$} & \multicolumn{2}{|c|}{ ЕСHO } & \multicolumn{2}{|c|}{ TOREX } \\
\hline & $\begin{array}{r}P S N \\
R(d B)\end{array}$ & $\begin{array}{c}\text { MSS } \\
\text { IM }\end{array}$ & $\begin{array}{c}P S N R \\
(d B)\end{array}$ & $\begin{array}{c}\text { MSSI } \\
M\end{array}$ & $\begin{array}{c}P S N R \\
(d B)\end{array}$ & $\begin{array}{c}\text { MSSI } \\
M\end{array}$ \\
\hline 0.0 & 29.70 & 0.81 & 28.67 & 0.776 & 32.51 & 0.810 \\
\hline 6 & 23 & 09 & 89 & 6 & 78 & 6 \\
\hline 0.0 & 27.78 & 0.77 & 27.19 & 0.730 & 31.05 & 0.768 \\
\hline 8 & 28 & 02 & 03 & 3 & 77 & 3 \\
\hline 0.1 & 26.05 & 0.73 & $\begin{array}{c}26.05 \\
50\end{array}$ & 0.688 & 29.89 & 0.735 \\
\hline 0.4 & $\begin{array}{c}17.61 \\
57\end{array}$ & $\begin{array}{c}0.48 \\
42\end{array}$ & $\begin{array}{c}19.27 \\
40\end{array}$ & $\begin{array}{c}0.468 \\
3\end{array}$ & $\begin{array}{c}21.38 \\
82\end{array}$ & $\begin{array}{c}0.549 \\
4\end{array}$ \\
\hline
\end{tabular}

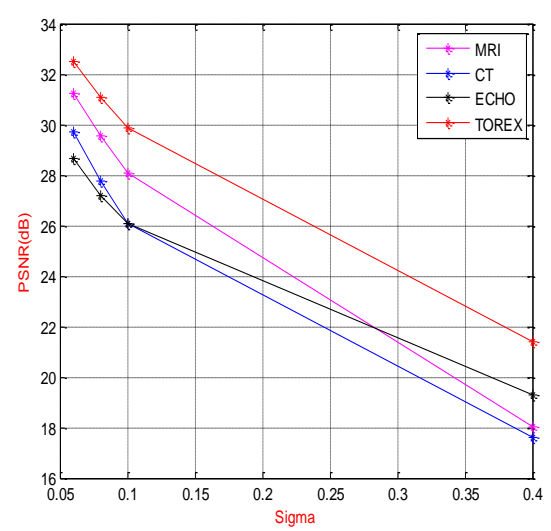

Figure 9. Comparisons between MRI, CT, ECHO and TOREX denoising image using bandelet transform for $\mathrm{T}=30$

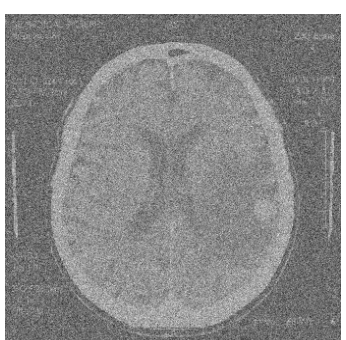

(a)

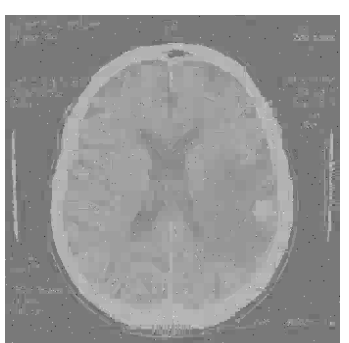

(b) 


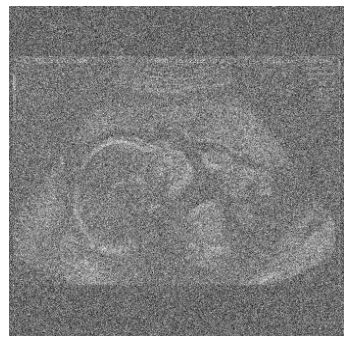

(c)

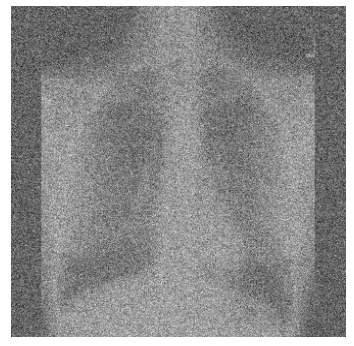

(e)

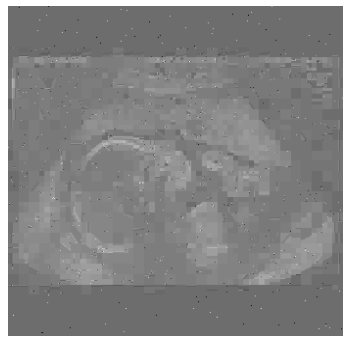

(d)

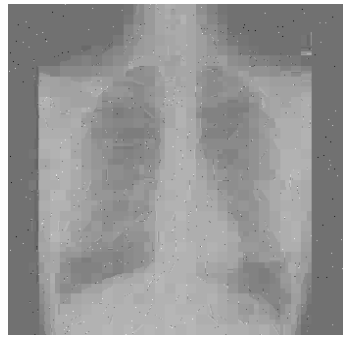

(f)
Figure 10. Denoising CT, ECHO and TOREX images for $\mathrm{T}=30$ :

(a) Noise CT image, (b) Denoising CT image, (c) Noise ECHO image, (d) Denoising ECHO image, (e) Noise TOREX image, (f) Denoising TOREX image

After comparing between the four curves In Fig 9. We can see that the bandelet transform is very effective and adaptable to TOREX image compared to MRI, CT images and ECHO this is justified by the values of PSNR.

In the following, we make a comparative study of filtering between bandelet, contourlet, curvelet, ridgelet transforms and wavelet in TOREX medical image. The results obtained are as follows:

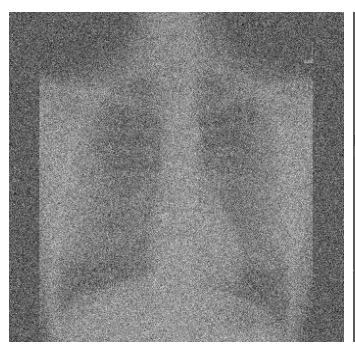

(a)

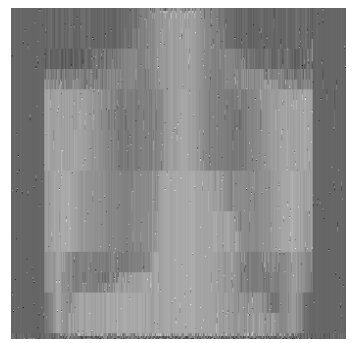

(c)

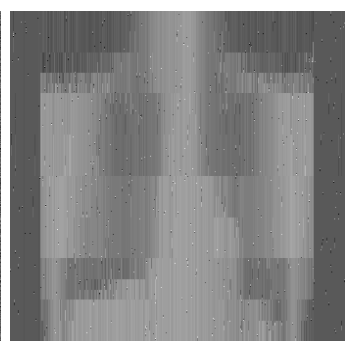

(b)

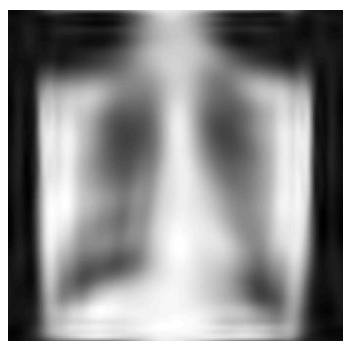

(d)

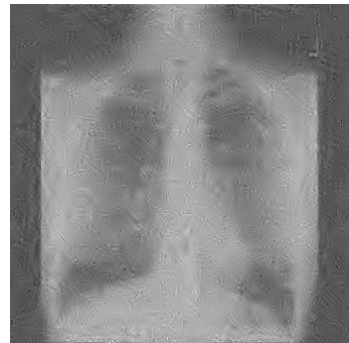

(e)

Figure 11. (a) Noise TOREX image, (b) Denoising TOREX image using wavelet transform, (c) Denoising TOREX image using ridgelet transform, (d) Denoising TOREX image using curvelet transform, (e) Denoising TOREX image using contourlet transform.

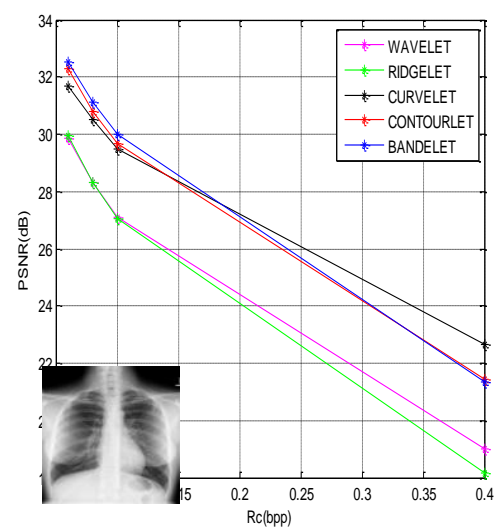

Figure 12. Comparisons between bandelet, contourlet, curvelet, ridgelet and wavelet transform for TOREX image

From Fig 12, we clearly perceive the importance of the bandelet transform compared with wavelet, ridgelet, curvelet transform and contourlet; this is proven with the PSNR values.

Fig 13 shown correlation coefficients results for a five transforms types used in de-noising domain to detect changes in an image.

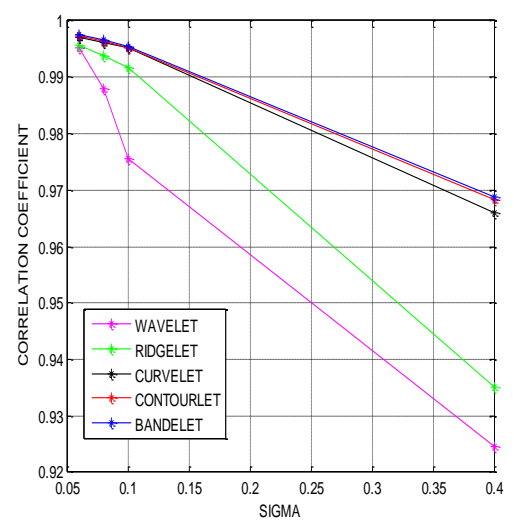

Figure. 13 Comparisons between correlation coefficient for a five wavelet transforms 
From the figure above we can say that the correlation coefficient from bandelet transform gives better results compared to other types of transforms.

After showing the performance of the de-noising medical images, we apply our algorithm to the denoising satellite images. We are interested in this application by the satellite image gray scale coded on 8 bpp of size $512 \times 512$.

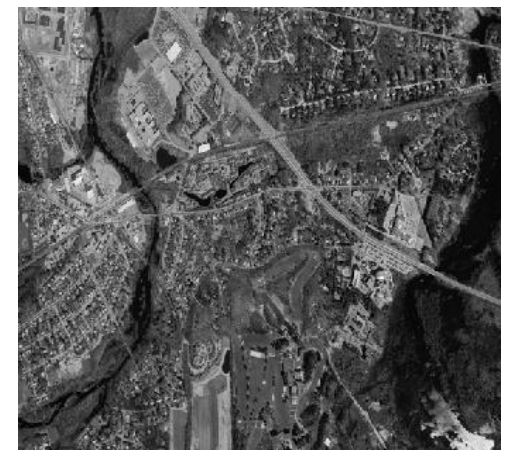

Figure 14. SATELLITE image

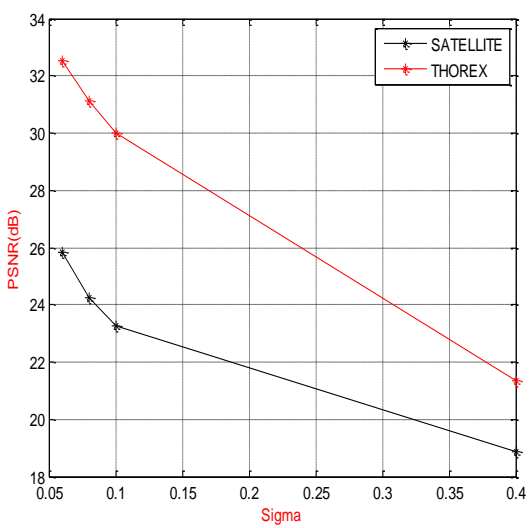

Figure 15. Comparisons between TOREX and SATELLITE denoising image using bandelet transform for $\mathrm{T}=30$

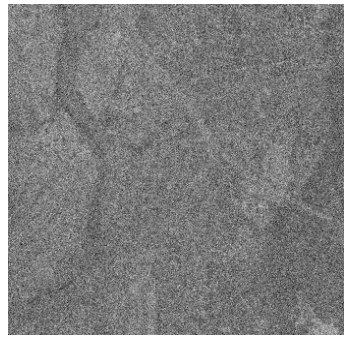

(a)

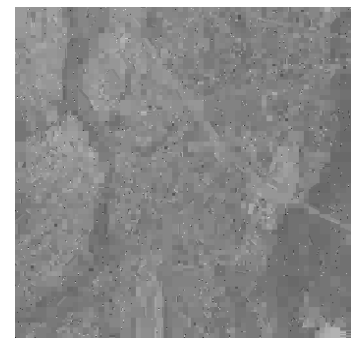

(b)
Figure. 16 Denoising SATELLITE for $\mathrm{T}=30$ :

(a) Noise SATELLITE image, (b) De-noising SATELLITE image.

From Fig 15, we see that the gain between satellite image and medical is very important, which shows the effectiveness of the de-noising image using bandelet transform for medical image (TOREX).

\section{VI.CONCLUSION}

In this paper, we presented a new de-noising algorithm using a new transform used in processing image, which is called bandelets transform. The proposed method was applied for comparison between two type image (medical and satellite) of size $512 \times 512 \times 8$ pixels contaminated by random noise. The comparison between different image using bandelet transform allows us to check the superiority of the bandelet transform in medical image which's justified by the PSNR and MSSIM highest values. Moreover, the bandelet transform provides compact representation and a major improvement compared to other classical transforms. We can assure that the bandelet transform outperforms the wavelet, the ridgelet, and the curvelet transform in term of PSNR values and visual quality which is the most pleasant among the all. The performance of the de-noising algorithm using the bandelet transform also performs well even in the cases where we have images with very high frequencies.

In the future we are going to work into defining a strategy to exploit the bandelet transform for image compression.

\section{REFERENCES}

[1] E.J.Candes and D. L.Donoho, "Ridgelets: A key to higherdimensional intermittency", Philosophical Trans of the Royal Society of London Series A, vol.357, pp.2495-2509, 1999.

[2] Minh N. Do and Martin Vetterli, "Image Demising using Orthonormal Finite Ridgelet Transform PTOCS. PIE, Wavelet Applications in Signal and Image Processing VIII", Akrain hldroubi; Andrew F. Laine; Michael A. Unser; Eds. Vol. 41 19, p. 831-842, Dec. 2000.

[3] E. Le Pennec et S. Mallat, "Représentation d'images par bandelettes et application à la compression", Dans GRETSI, Toulouse, Septembre 2001.

[4] E. Le Pennec, "Bandelettes et représentation géométrique des images", Thèse de Doctorat, Ecole Polytechnique, 19 décembre 2002.

[5] V. Chappelier, "Codage progressif d'images par ondelettes orientées", Thèse de Doctorat, Université Rennes 1, 15 décembre 2005.

[6] Jian-Jiun Ding, "Time Frequency Analysis Tutorial", R99942057.

[7] Xiaokai Wang, Jinghuai Gao, "Image Denoising Method Based on Nonsubsampled Contourlet Transform and Bandelet Transform," The 1st International Conference on Information Science and Engineering (ICISE), Institute of Wave and Information, Xi'an Jiaotong University, Xi'an, China, 2009.

[8] Hong Han,Xing Wu, "Research of Method in Human Detection Based on Bandelet Transform", Laboratory of Intelligent Perception and Image 
Understanding of Ministry of Education of China, Vol. 74951S, 2009.

[9] Ruihua Liang, Lizhi Cheng, Zhicheng Zhu, Bo Chen, "Image coding based on second generation bandelet transform", Modern electronics technique, 61-65 (2007).

[10] E. Le Pennec, S. Mallat, "Bandelettes et représentation géométrique des images", CMAP / école Polytechnique 91128 PALAISEAU - France.

[11] E. Le Pennec, S. Mallat, "Bandelet Representations For Image Compression", 0-7803-6725-1/01. IEEE. 2001.

[12] W.S. Geisler, M.S. Banks, "Visual Performance, Handbook of Optics", Vol. 1, McGraw-Hill, NY, USA, 1995.

[13] A.B. Watson, L.B. Kreslake, "Measurement of Visual Impairment Scales for Digital Video, Human Vision and Electronic Imaging", Conference, San Jose, CA, USA, SPIE Vol. 4299, Jan. 2001, 2001, pp. $79-89$.

[14] Eugene K. Yen and Roger G. Johnston, "The Ineffectiveness of the Correlation Coefficient for Image Comparisons", Vulnerability Assessment Team, Los Alamos National Laboratory, MS J565, Los Alamos, New Mexico 87545.

[15] www.GE Medical System.com (database).

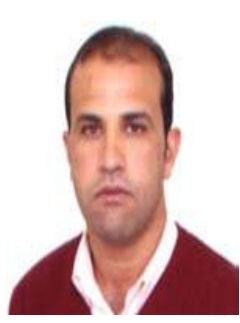

Mohammed BELADGHAM was born in Tlemcen, Algeria; he's received the electrical engineering diploma from university of Tlemcen, Algeria, and then a Magister in signals and systems from university of Tlemcen, Algeria and the Ph.D. degree in Electronics from the University of Tlemcen (Algeria), in 2012. His research interests are Image processing, Medical image compression, wavelets transform and optimal encoder.

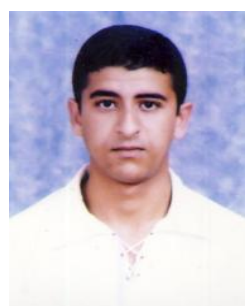

Yassine HABCHI was born in Mechria, Algeria, received his Bachelor (2005) and Engineers (2010) degree in Electrical Engineering from Saïda University in Algeria, and his Magister (2013) degree in Electronic from university of Bechar, Algeria. His current research interest includes Image processing, Medical image compression, wavelets transform and optimal encoder.

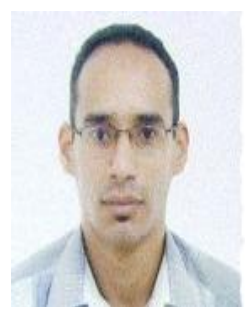

MOULAY LAKHDAR I got my Engineering Degree in Telecommunication in 2000 at the Institute of Telecommunications in Oran. Magister was my second degree in Signal and telecom at Djillali LIABES university of Sidi Bel Abbes in 2003. From 2004 to this day I work in the Bechar University as lecturer. Since May 2009, I graduated PhD Es Sciences at the of Sidi Bel Abbes. I do my research at the Bechar University and Communications, Architecture and Media Laboratory (CAMR) (Djillali LIABES University). His research interests are Image transmission, Image processing, and digital transmission performances.

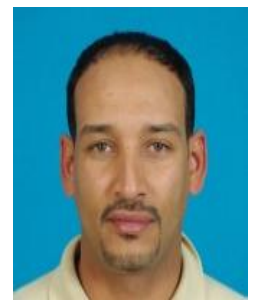

Abdesselam Bassou was born in Bechar, Algeria. He received the Dipl.El.-Ing. Degree from the University of Tlemcen, Algeria in 1997, his Master from the University of Sidi Bel Abbes, Algeria in 2000, and his Doctoral degree Es Abdesselam Bassou was born in Bechar, Algeria. He received the Dipl.El.-Ing. Degree from the University of Tlemcen, Algeria in 1997, his Master from the University of Sidi Bel Abbes, Algeria in 2000, and his Doctoral degree Es Science from the University of Sidi Bel Abbes, Algeria in 2006. Actually, $\mathrm{He}$ is an Associate Professor at University of Bechar, Algeria. His main interests are digital signal processing, turbo encoding schemes and iterative decoding over fading channels, and channel equalization.

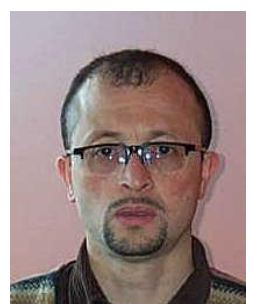

AbdelmalikTALEB-AHMED Was born in Roubaix, France, in 1962. He received a post graduate degree and a $\mathrm{Ph}$. D. in Electronics and Microwaves from the University of Lille1 in 1988 and 1992. From 1992 to 2004, He was an Associate Professor at the University of Littoral, Calais. Since 2004, He is currently a Professor at the University of Valenciennes in the department GE2I, and does his research at the LAMIH FRE CNRS 3304 UVHC, His research interests includes signal and image processing. Image segmentation, Prior knowledge integration in image analysis, Partial Differential Equations and Variational Methods in image analysis, Image compression, Multimodal signal processing, Medical image analysis, including multimodal image registration, etc.. 